\title{
COMMENTS ON THE EFFECT \\ OF $\gamma$-IRRADIATION ON POSITRONIUM FORMATION IN POLYMERS AT LOW TEMPERATURES
}

\author{
V.P. Shantarovich ${ }^{a}$, T. $\operatorname{Hirade}^{b}$, I.B. Kevdina ${ }^{a}$, V.W. Gustov ${ }^{a}$ \\ AND E.F. OLEINIK ${ }^{a}$ \\ ${ }^{a}$ Semenov Institute of Chemical Physics, Russian Academy of Sciences \\ 4 Kosygin str., Moscow, 117334, Russia \\ ${ }^{b}$ Department of Material Science, Japan Atomic Energy Research Institute \\ 319-1195, Tokai, Japan
}

\begin{abstract}
Quantitative description of the effect of $\gamma$-irradiation of PE and PMMA on positronium formation on the trapped electrons at low temperatures is suggested. The consideration shows that, at least in these substances, the number of the trapping centers is probably enough to localize all ortho-positronium, and therefore the intensity of the long-lived positronium component is dependent mostly on the probability of Ps formation but not on the number of the trapping centers.
\end{abstract}

PACS numbers: $78.70 . \mathrm{Bj}$

\section{Introduction}

Thus far, there is a problem: has the long-lived Ps component $I_{3}$ something to do with the number of $o$-Ps trapping sites or is this number so high that $I_{3}$ is dependent only on the fraction of positrons $Q$ which form Ps in a system [1-5]? The last alternative $[4,5]$ was proposed recently and it suggests that the $o$-Ps trapping rate $\nu_{\mathrm{T}}$ is much higher than the annihilation rate of free $o-\mathrm{Ps} \lambda_{\mathrm{f}}, \nu_{\mathrm{T}} \gg \lambda_{\mathrm{f}}$. It is likely that a quantitative description of the results [5] on Ps formation on the trapped electrons (their concentration was measured using ESR) in $\gamma$-irradiated PE and PMMA gives the first chance to obtain straight argument in favor of the second alternative. Some of our data on thermostimulated luminescence (TSL) glow support the suggested mechanism of Ps formation on the trapped electrons. 


\section{Experimental}

We describe analytically the experimental results [5] consisting in observations of additional $o$-Ps formation $\Delta I_{3}$ (relative to $I_{3}=3 Q / 4$ for a non-irradiated sample) in PMMA and high density PE due to low-temperature $\gamma$-irradiation by the ${ }^{60}$ Co source (Figs. 1, 2). Figure 3 demonstrates TSL glow curves for high density PE (curve A) and PMMA (curve B) measured in this work. Figure 4 shows our TSL results for the two copolymers of the same chemical composition but with different thermal history. One of them was original copolymer poly (methyl methacrylate-co-methacrylic acid) with molar feed composition of monomer $70 / 30$ (curve 1). The second one was annealed at $140^{\circ} \mathrm{C}$ for 0.5 hour (curve 2).

\section{Discussion}

The increase $\Delta I_{3}$ of $P$ s formation in some polymers because of $\gamma$-irradiation (either by ${ }^{22} \mathrm{Na}$ source during the experiment or after the exposure to $\gamma$-rays from ${ }^{60} \mathrm{Co}$ source) at low temperatures was observed recently by many investigators. The authors of [5] suggested a qualitative explanation, supposing additional Ps formation on weakly bound electrons (trapped electrons) created by $\gamma$-irradiation. Essential progress in this study was obtained due to complementary ESR measurements of the trapped electrons concentration $N_{\mathrm{e}}$. We propose here a quantitative description of the model [5]. The variation $\Delta I_{3}$ is found from the following equations describing Ps formation (rate $\nu_{\text {form }}$ ) and subsequent Ps trapping (rate $\nu_{\mathrm{T}}$ ) by the trapping centers (concentration $N_{3}$ ) in the free volume space:

$$
\begin{array}{ll}
\mathrm{d} P_{\mathrm{f}}^{+} / \mathrm{d} t=-\left(\lambda_{\mathrm{f}}^{+}+\nu_{\text {form }}\right) P_{\mathrm{f}}^{+}, & P_{\mathrm{f}}^{+}(0)=1-Q, \\
\mathrm{~d} P_{\mathrm{f}}^{\mathrm{Ps}} / \mathrm{d} t=(3 / 4) \nu_{\text {form }} P_{\mathrm{f}}^{+}-\left(\lambda_{\mathrm{f}}^{\mathrm{Ps}}+\nu_{\mathrm{T}}\right) P_{\mathrm{f}}^{\mathrm{Ps}}, & P_{\mathrm{f}}^{\mathrm{Ps}}(0)=0, \\
\mathrm{~d} P_{\mathrm{T}}^{\mathrm{Ps}} / \mathrm{d} t=\nu_{\mathrm{T}} P_{\mathrm{f}}^{\mathrm{Ps}}-\gamma_{3} P_{\mathrm{T}}^{\mathrm{Ps}}, & P_{\mathrm{T}}^{\mathrm{Ps}}(0)=0 .
\end{array}
$$

$Q$ is the fraction of positrons which form Ps without $\gamma$-irradiation; the rest of the positrons $(1-Q)$ escape from the spur and can try to form Ps on the trapped electrons. We shall use $P_{\mathrm{f}}^{+}$as the probability to find free $e^{+}, \lambda_{\mathrm{f}}^{+}$as the free $e^{+}$ annihilation rate, and $\lambda_{\mathrm{f}}^{\mathrm{Ps}}$ as the annihilation rate of free («pre-localized») Ps; we suppose that $\lambda_{\mathrm{f}}^{+} \cong \lambda_{\mathrm{f}}^{\mathrm{Ps}} \equiv \lambda_{\mathrm{f}}$. Finally, $P_{\mathrm{f}}^{\mathrm{Ps}}$ and $P_{\mathrm{T}}^{\mathrm{Ps}}$ are the probabilities to find free and trapped $o$-Ps, correspondingly. Obviously, o-Ps lifetime in the trapping center is shortened compared to its intrinsic lifetime and equal to $\tau_{3}=1 / \gamma_{3}$. The intensity of this component is denoted as $I_{3}$. The solution of Eqs. (1) gives an increase in $I_{3}, \Delta I_{3}$, because of additional Ps formation on the trapped electrons

$$
\begin{aligned}
\Delta I_{3} & =(3 / 4)(1-Q) \nu_{\text {form }}\left(\lambda_{\mathrm{f}}+\nu_{\text {form }}-\gamma_{3}\right) \nu_{\mathrm{T}}\left(\lambda_{\mathrm{f}}+\nu_{\mathrm{T}}-\gamma_{3}\right) \\
& =(3 / 4)(1-Q) A B,
\end{aligned}
$$

where $B=\gamma_{\mathrm{T}}\left(\lambda_{\mathrm{f}}+\nu_{\mathrm{T}}-\gamma_{3}\right)^{-1}, \nu_{\mathrm{form}}=4 \pi D_{\mathrm{f}}^{+} R N_{\mathrm{e}}=k_{\text {form }} N_{\mathrm{e}}$ and $\nu_{\mathrm{T}}=4 \pi D_{\mathrm{f}}^{\mathrm{Ps}} R_{3} N_{3} . D_{\mathrm{f}}^{+}$and $D_{\mathrm{f}}^{\mathrm{Ps}}$ are diffusion coefficients of free $e^{+}$and Ps, correspondingly; $R$ is an effective radius for interaction of $e^{+}$and trapped electron, and $R_{3}$ describes an effective size of a trapping center. 
It becomes obvious that fitting of Eq. (2) to experimental points for PE is possible only with $k_{\text {form }}(\mathrm{PE})=\nu_{\text {form }} / N_{\mathrm{e}}=10^{-7} \mathrm{~cm}^{3} / \mathrm{s}$ and $\nu_{\mathrm{T}} \gg \lambda_{\mathrm{f}}$, i.e. $B=1$ (Fig. 1). A description of the PMMA results for the highest $N_{\mathrm{e}}=2 \times 10^{17} \mathrm{~cm}^{-3}[5]$ is possible by suggesting the two following alternatives:

(a) $\nu_{\mathrm{T}} \gg \lambda_{\mathrm{f}}, B=1$, and $k_{\text {form }}(\mathrm{PMMA})=(1 / 50) k_{\text {form }}(\mathrm{PE})=0.24 \times 10^{-8} \mathrm{~cm}^{3} / \mathrm{s}$. The last ratio probably means that $D_{\mathrm{f}}^{+}(\mathrm{PMMA})=(1 / 50) D_{\mathrm{f}}^{+}(\mathrm{PE})$. The diffusion coefficient for $\mathrm{PE}$ is known to be about $0.1 \mathrm{~cm}^{2} / \mathrm{s}$, however $D_{\mathrm{f}}^{+}(\mathrm{PMMA})$ is unknown.

(b) $\nu_{\mathrm{T}}$ and $\lambda_{\mathrm{f}}$ are comparable. Fitting to the highest value of $\Delta I_{3}$ gives the probability of Ps trapping $B=0.2$, if the rate constant of Ps formation on the trapped electrons in PE and PMMA are roughly the same: $k_{\text {form }}(\mathrm{PMMA})=$ $k_{\text {form }}(\mathrm{PE})=10^{-7} \mathrm{~cm}^{3} / \mathrm{s}$.

A comparison of calculated dependences for the two cases mentioned above is given in Fig. 2.

We have to conclude that for PMMA, just as for PE, the alternative (a) gives a much better description of the whole set of points [5] than the alternative (b). And this is the first direct argument in favor of the point of view that in such polymers as PE and PMMA all ortho-Ps atoms are probably trapped before annihilation $(B=1)$. It means that in such cases $I_{3}$ is dependent mostly on the probability of Ps formation in a substance $Q$ but not on the number of the trapping sites $N_{3}$, since the number of Ps traps is too high $\left(\nu_{\mathrm{T}} \gg \lambda_{\mathrm{f}}\right)$. Further experiments of this type could be useful. In the scope of the further study of the mechanism of Ps formation on the trapped electrons we suggest a comparison of the observed effect with results on TSL glow for the same samples at low tempera-

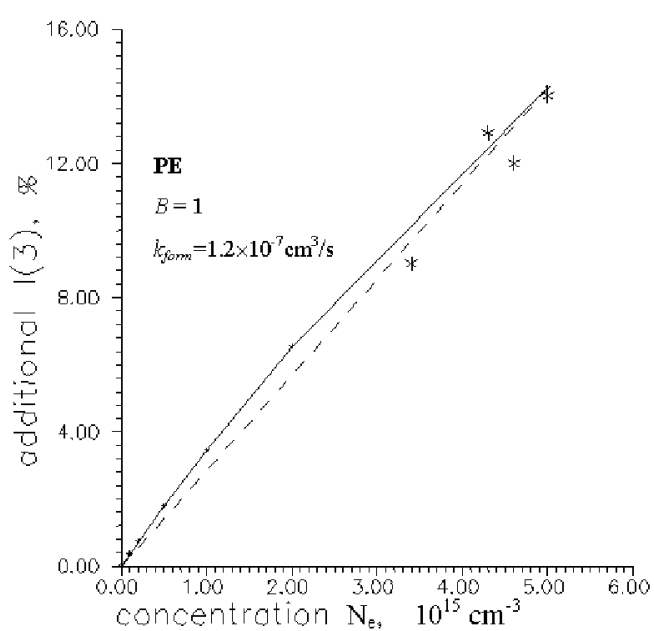

Fig. 1. Additional intensity $\Delta I_{3}$ of the longest lifetime component in $\mathrm{PE}$ as a function of the trapped electron density [5]. The dependence is described (solid line) by Eq. (2) with $k_{\text {form }}(\mathrm{PE})=1.2 \times 10^{-7} \mathrm{~cm}^{3} / \mathrm{s}$ and $B=1$. The dashed line here and in Fig. 2 represents the linear dependence for comparison. 


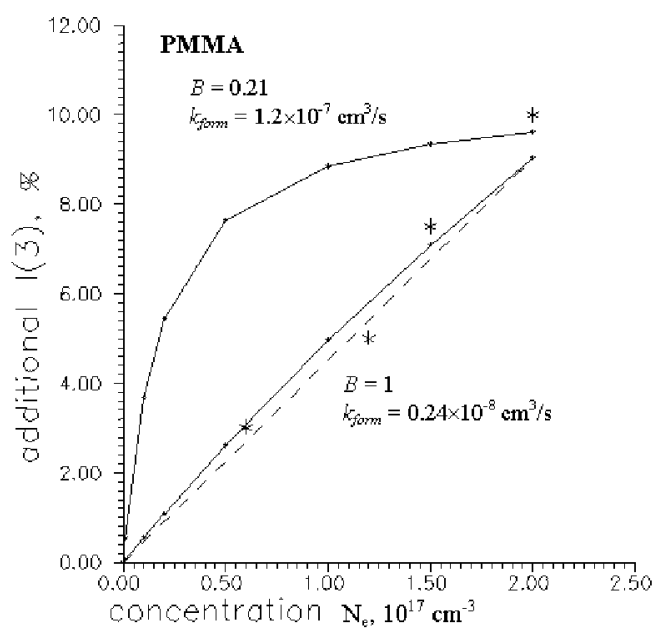

Fig. 2. Additional long-lived component intensity in PMMA as a function of the trapped electron density [5]. Solid curves correspond to different sets of parameters in Eq. (2): $k_{\text {form }}(\mathrm{PMMA})=k_{\text {form }}(\mathrm{PE})=1.2 \times 10^{-7} \mathrm{~cm}^{3} / \mathrm{s}, B=0.21$ or $k_{\text {form }}(\mathrm{PMMA})=$ $(1 / 50) k_{\text {form }}(\mathrm{PE})=0.24 \times 10^{-8} \mathrm{~cm}^{3} / \mathrm{s}, B=1$.

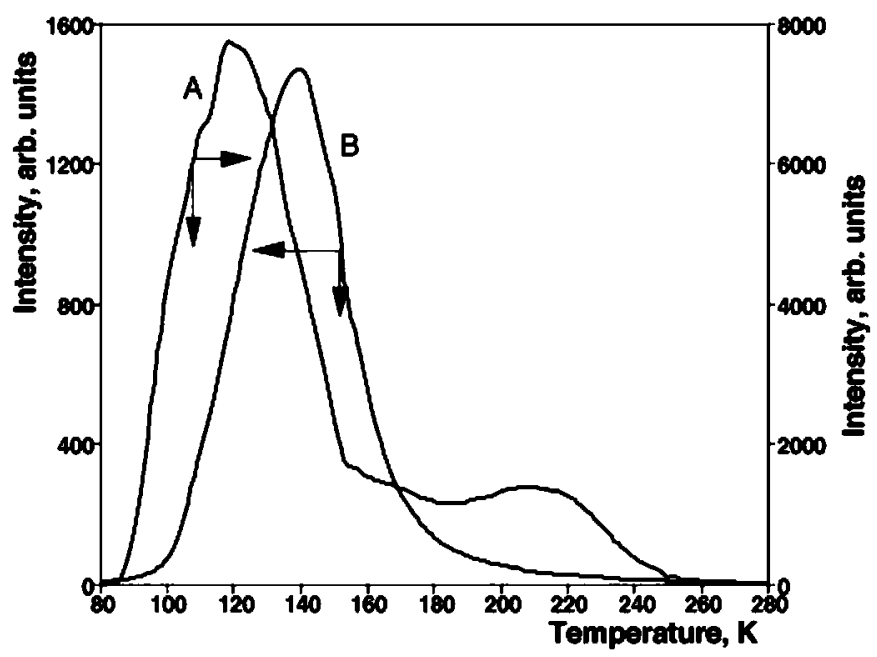

Fig. 3. TSL glow curves for PE (A) and PMMA (B). All curves in this figure and in Fig. 4 are represented in the same relative units.

ture (Figs. 3, 4). Though irradiation dozes normally used in TSL glow experiments are slightly higher $(5-10 \mathrm{kGy})$ than those in PAL study [5], it seems obvious that the mechanisms of the two processes have much in common. Actually, the electrons trapped at low temperature are responsible for the both effects. Both effects can be essentially reduced by bleaching. It is obvious from Fig. 3 that TSL glow 


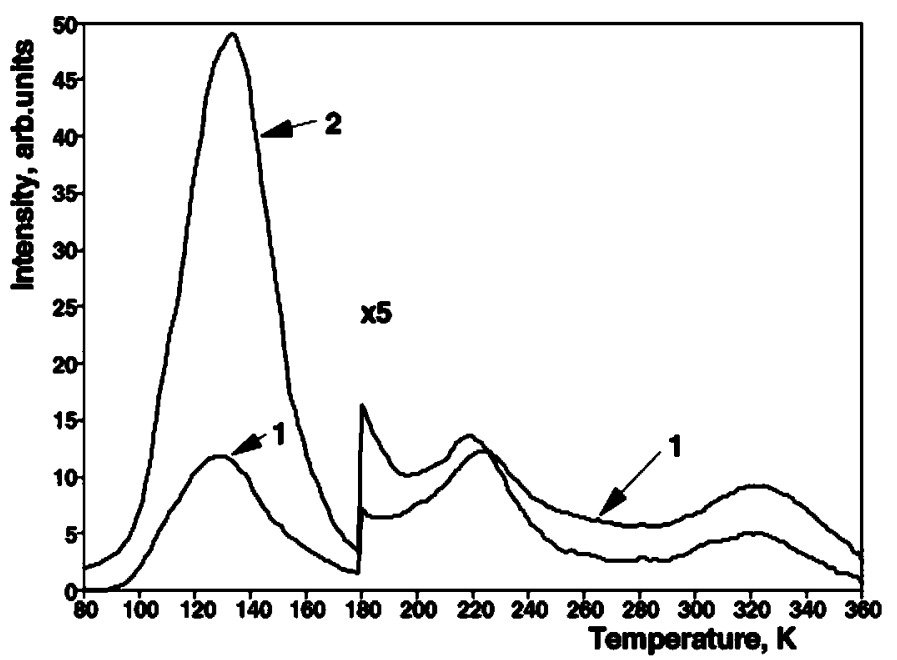

Fig. 4. TSL glow curves for original (curve 1) and annealed (curve 2) MMA-MAC (70/30) copolymers.

of PE is much more intensive than that of PMMA for $\gamma$-irradiation with the same dozes (just as the effect of Ps formation on the trapped electrons). A comparison of TSL glow (Fig. 4) for the samples having the same chemical composition but different thermal history (see Sec. 2) and Ps yield at given temperature and dozes would be of special interest.

\section{Acknowledgments}

This work was supported by the Russian Foundation for Basic Research, project No.98-03-32859, IR 97-1525, and by the INTAS grant No. 97-1936.

\section{References}

[1] W. Brandt, S. Berko, W.W. Walker, Phys. Rev. 120, 1229 (1960).

[2] Y.C. Jean, Q. Deng, T.T. Nguen, Macromol. 28, 8840 (1995).

[3] V.P. Shantarovich, V.I. Goldanskii, Hyperfine Interact. 116, 67 (1998).

[4] C. Dauve, G. Consolati, J. Kansy, E. Van Waeyenberge, Phys. Lett. A 238, 379 (1998).

[5] T. Hirade, F.H.J. Maurer, M. Eldrup, Radiat. Phys. Chem. 58, 465 (2000). 